\title{
Guest Editorial: Selected Papers from the INFN- LNF Conference on Nanoscience and Nanotechnology
}

\author{
Stefano Bellucci \\ Istituto Nazionale di Fisica Nucleare \\ Laboratori Nazionali di Frascati \\ Via E. Fermi 40, 00044 Frascati, Italy \\ bellucci@lnf.infn.it
}

The fast growing area of nanoscience has attracted the attention of the scientific community, as well as society at large, especially for promising nanotechnological applications. The Nanoscience \& Nanotechnology 2008 (n\&n2008) conference held at Laboratori Nazionali di Frascati in Frascati, Italy (see http://www.lnf.infn.it/conference/nn2008/) aimed to assess the current state of the art and stimulate research networking, and was held under the patronage of INFN (Italian Institute for Nuclear Physics), with the generous sponsorship by LOT Oriel Italy and Horiba Jobin Yvon.

Such yearly meetings took place earlier within the Nanotubes \& Nanostructures $(\mathrm{N} \& N)$ School and Workshop series [1,2], including a conference in Cagliari in 2000 and conferences in Frascati from 2001-2004.

More recently, the focus has shifted from the study of nanomaterials to the, by now, mature field of nanoscience and the corresponding innovative technologies in the most promising fields of application, hence yielding the Nanoscience \& Nanotechnology (n\&n) series [3-5]:

Selected, refereed papers, based on conference original presentations and follow-up discussions, are published in this special section of the SPIE Journal of Nanophotonics. For volumes including lectures given at earlier editions of the school in 2006 and 2007, see Refs. 6 and 7.

\section{References}

[1] S. Bellucci, Ed., Proc. School and Workshop on Nanotubes \& Nanostructures 2000, Santa Margherita di Pula (Cagliari), Italy, 24 September to 4 October 2000, Italian Physical Society, Bologna, Italy (2001).

[2] M. De Crescenzi and S. Bellucci, Eds., J. Phys.: Condens. Matter 15(34) (2003).

[3] S. Bellucci, Ed., J. Phys.: Condens. Matter 18(33), S1967-S2238 (2006).

[4] S. Bellucci, Ed., J. Phys.: Condens. Matter 19(39), 390301-395024 (2007).

[5] S. Bellucci, Ed., J. Phys.: Condens. Matter 20(47), 470301, 474201-474214 (2008).

[6] S. Bellucci, Ed., "Nanoparticles and Nanodevices in Biological Applications. The INFN Lectures - Vol I," Lecture Notes in Nanoscale Science and Technology, Vol. 4, pp. 1-198, Springer, Heidelberg, Germany (2009).

[7] S. Bellucci, Ed., "Transport in nanoparticles and nanodevice. The INFN lectures Vol II," Lecture Notes in Nanoscale Science and Technology, Springer, Heidelberg, Germany (in press). 